\title{
A CASE OF SURGICAL EMPHYSEMA OCCURRING SPONTANEOUSLY IN LABOUR
}

\author{
P. CORKERY, M.B., CH.B. ${ }^{*}$
}

A young CANAdian girl of Scottish extraction, mesomorph in build, 120 pounds at full term, a primigravid aged nineteen, had been in labour for ten hours, and was near delivery. Meperidine $75 \mathrm{mg}$ given four hours before was the only sedation. An outlet forceps assisted delivery was intended.

The patient was exhorted to push with the last few contractions prior to the induction of general anaesthesia, and she cooperated with all her strength; pushing against a closed glottis.

A semi-closed system using nitrous oxide and oxygen, 5 litres and 2 litres with trichlorethylene in a Rowbotham vaporizer, was used to produce rapid analgesia and later anaesthesia. Her breathing became snoring in character. A soft lubricated nasopharyngeal airway was inserted gently without difficulty, and spontaneous ventilation continued freely. No positive pressure was applied to the airway at any time.

Before the anaesthesia was terminated, swelling was noted in the cervical

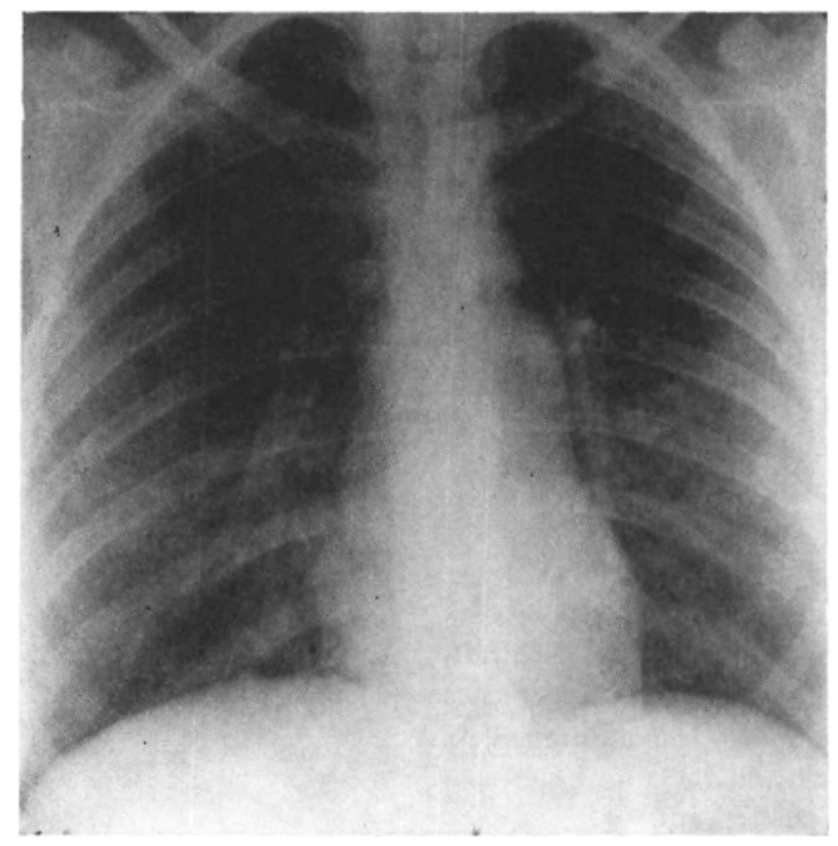

Figure 1

'Department of Anaesthesia, Royal Alexandra Hospital, Edmonton, Alberta.

643

Can. Anaes. Soc. J., vol. 17, no. 6, November 1970 
region, bilaterally above the clavicles. It had the typical crepitus of surgical emphysema. There was no respiratory distress, and no sign of mediastinal crunch or pneumothorax was elicited. Anaesthesia was discontinued when the episiotomy was repaired. Vital signs remained stable. Roentgenograph (Figure 1) showed gas in the mediastinum, and in the subcutaneous tissues in the neck.

The patient awoke, and complained of "a feeling of swollen glands in the neck". There was no history of previous pulmonary disease or incident of pneumothorax.

Equipment for the rapid relief of possible tension pneumothorax was kept by her bedside, but the emphysema regressed without incident over the next five days.

Because in this case a nasopharyngeal airway was used, it raises the possibility of iatrogenic trauma to the nasopharynx and the consequent passage of air into the subcutaneous tissues. However, there was no evidence of trauma. There was no slight trace of blood on the tube, in the pharynx, or in the nostril. The tube slid easily in the first nostril attempted. There was no respiratory distress or obstructed breathing, or positive pressure used afterwards, and because of these factors the above possibility seems unlikely.

\section{AETiology of Ectopic GASES}

From the point of view of the practising anaesthetist it is alarming to read of the number of different ways our gaseous environment can get into the wrong compartment in our bodies. Some of these are listed below:

1. Trauma

a) Injury to the chest with or without broken ribs

b) Operation on the chest (especially induction of pneumothorax)

-Thoracentesis

-Brachial plexus block (supraclavicular)

-Radical mastectomy

-Arteriogram (supraclavicular)

- Liver or spleen needle biopsy

-Intercostal nerve block

- Trans-lumbar aortogram

-Bronchoscopy

-Radical neck surgery

-Thyroidectomy

-Parathyroid surgery

-Paravertebral cardiac catheterization

2. Increased Airway Pressure

a) Increase of intra-pulmonary pressure

-Explosions

-Heavy lifting

-Straining at stool

-Childbirth

-Attempts to resuscitate the newborn 
-Accidents with SCUBA and other breathing equipment

-Anaesthesia (if too high positive pressure is used, or if the air passages are damaged by the tube)

b) Increased airway pressure with occlusion, partial or complete, of the trachea or bronchi, especially if accompanied by cough or buck

-Anaesthesia (if too narrow, or kinked tube, or other defect)

-Asthma

-Bronchitis

-F.B. in bronchus

-Pneumonia

-Whooping cough

3. Spontaneous rupture of alveoli (without the increased pressure)

This can happen where there is no obvious disease process, but also in:

-Rupture of emphysematous bulla, or,

- T.B. focus, or,

-Other inflammatory or malignant disease process

\section{Rarer Causes}

-Following ruptured oesophagus

- Air from compressed air dental drill (Charlebois ${ }^{1}$ )

- Barium enema

-Sigmoidoscopy

-Gas gangrene

-Following irrigation of wounds by hydrogen peroxide

-In limb after penetrating wound. Movement aspirates the air and moves it along the subcutaneous planes.

-Magnesium alloys in industry going subcutaneously through trivial wounds and liberating hydrogen later.

-Diabetic Hyperpnoea

Sivak $^{2}$ reported a case of surgical emphysema following fulguration of the bladder. Gases escaped through an iatrogenic bladder aperture below the peritoneal reflection. The gases were tracked to the retroperitoneal space, finally appearing in the neck, face, and axilla. Sivak was of the opinion that the gases were a by-product of the fulguration. In addition, air bubbles entering during attempted bladder washout in the presence of an unrecognized bladder perforation, can also produce surgical emphysema.

\section{InCidence of SPontaneous Emphysema in Labour}

The sudden onset of the phenomenon in the present case was alarming, despite the patient's good general condition. Gordon ${ }^{3}$ reported two cases, and collected 130 others in the literature. By $1963,{ }^{4} 187$ cases had been reported. It is most common in the healthy young primigravid, in the course of a strenuous first or second stage of labour. A few cases have been noted for the first time post partum.

\section{The Mechanism}

The most common mechanism producing the phenomenon is as follows: A large inspiration produces a large lung volume. The glottis is closed, and the cavity 
of the chest is compressed by terrific straining effort. At a critical pressure, the parenchyma of the lung gives way, and if the pressure continues, the air from the alveolus goes either sub-pleurally, or alongside the vessels into the mediastinum, then along the vessels of the trachea into the neck, and via nerve and vessel pathways into cervical cellular tissues.

Gordon thought that pneumothorax accompanying the condition was rare, but the investigations of Kelman, ${ }^{5}$ Keis, ${ }^{6}$ Macklin and Macklin ${ }^{7}$ are informative. Antoni ${ }^{8}$ repeated Keis' experiment of inflating the mediastinum in a corpse. A pneumothorax was produced. The air entered the pleural cavity just above the hilum of the lung. The opening is valvular, making the pneumothorax the tension type in one or both sides. Movement in the reverse direction, i.e. pleura to mediastinum, does not occur.

\section{The Fascial Layers and Tracking}

The cervical fascia is in two divisions, superficial and deep. The deep fascia is further divided into three layers:

1. Superficial

2. Middle: this pretracheal layer is continuous with the fibrous pericardium

3. The deep prevertebral layer is continuous with the endothoracic fascia

The space between middle and deep layers contains the trachea, oesophagus, and thyroid gland, and communicates freely with the superior mediastinum.

If the respiratory mucosa is damaged, air can be forced into the pretracheal space on expiration, and from here it may be sucked down into the mediastinum on inspiration.

This type of pneumomediastinum can occur if the skin is sutured too tightly around a tracheostomy cannula. The tension pneumomediastinum may cause cardiac tamponade and will impede flow in the great vessels.

\section{Sites of Break in the Respiratory Lining}

Air can move into the subcutaneous tissues by many routes. Nicaise ${ }^{9}$ believed the posterior weaker portion of the trachea could herniate and rupture as in whooping cough.

Watson $^{10}$ and Volpito ${ }^{11}$ showed that an injection of air through the buccal mucosa at the piriform fossa level, soon spreads out into the tissues of the face, neck and chest.

Lacrimal bone fracture, and injury along the course of the tear-duct are possible sites of entry.

Gas under pressure in the mediastinum may track:

1. Via rupture of the mediastinal pleura to form a pneumothorax

2. Via dissection into neck, face, axilla and wall of thorax

3. Following the aorta and oesophagus into the retro-peritoneal area.

Lightman and Lincoln ${ }^{12}$ reported a case of blunt injury to the chest wall, with extreme respiratory difficulty. There was no apparent or $\mathrm{x}$-ray evidence of pneumothorax or pneumomediastinum. The major trouble was interstitial pulmonary emphysema. A stormy course was pursued, until with the help of IPPV with high oxygen concentration, the gas tracked into the subcutaneous tissue. The vital 
capacity improved; in other words, the surgical emphysema acted as a safety valve.

In the older series of Gordon, there were two deaths. The autopsy reported on one showed air subpleurally, but not generally in the lung interstitium.

\section{SIGNS AND SYMPTOMS}

In the present case, surgical emphysema was noted while the patient was under general anaesthesia, and her vital signs remained stable.

Two of Gordon's cases said that they felt "something tearing in the neck" before the emphysema appeared. Chest pain, dyspnoea, "wheezy" respiration, diminution in vital capacity, tachycardia, cardiac arrhythmias, and hypotension may occur. There may also be dysphagia, hoarseness or aphonia, distant heart sounds, and there may be a tympanitic note on percussion over the mediastinum. Hammam's sign, a mediastinal "crunch" synchronous with systole, may be heard on auscultation. One patient was reported quite conscious of it.

\section{Treatment}

The situation could be urgent, brooking no delay for the luxury of radiological confirmation. Both sides of the chest may need aspiration. The airway must be cleared. Simple needle aspiration is not sufficient in some cases, and closed chest tube drainage, with under-water seal and suction, may be necessary, with frequent $\mathrm{x}$-ray follow-up, IPPV with a high oxygen concentration may be required.

\section{SUMMARY}

Another case of spontaneous surgical emphysema occurring during labour is presented. The mechanism of causation is discussed. The phenomenon can be sudden and alarming, but with methods of treatment available, the prognosis on the whole seems favourable.

\section{REFERENCES}

1. Charlesbois, P. A. Three Unusual Cases of Emphysema. Canad. Anaesth. Soc. J. 11: 104 (1964).

2. Sivak, B. J. Surgical Emphysema. Anaesthesia and Analgesia. 4: 415 (1964).

3. Gordon, C. A. Respiratory Emphysema in Labour. Amer. J. Obs. \& Gyn. 14: 633 (1927).

4. Brili, C. L.; Falsetti, H. L.; \& Lipton, H. P. Mediastinal Emphysema Complicating Labour. Amer. Review Resp. Disease. 94: 615 (1966).

5. Kelman, S. C. Experimental Emphysema. Archives Int. Medicine. 24: 332 (1919).

6. KeIs, J. Studies on the Cause of Mediastinal Emphysema and Pneumothorax in Goitre Operation. Munch. Med. Wschr. 91: 669 (1934).

7. Macklin, M. T. \& MACKLIN, C. C. Malignant Interstitial Emphysema of the Lung and Mediastinum as an Important Occult Complication in Many Respiratory Diseases and Other Conditions. Medicine. 23: 281 (1944).

8. Antoni, R. O. \& Ponka, J. L. The Hazard of Iatrongenic Pneumothorax. Surg., Gyn. Obst. 24: 13 (1961).

9. Nicaise, E. Rev. de Chir. 16:538 (1896).

10. Watson, D. B.M.J. 699 (1885).

11. Smith, Robent H.; Pool, Leland L.; \& Volpitto, Perry P. Subcutaneous Emphysema as a Complication of Intubation. Anesthesiology. 20: 714 (1959).

12. Lightman, D. J. \& Lincoln, J. C. R. Malignant Pulmonary Interstitial Emphysema. B.J.A. $39: 587$ ( 1967$)$. 